Short communication

\title{
MOLECULAR DETECTION OF CHLAMYDIA PSITTACI AND CHLAMYDIA FELIS IN HUMAN KERATO- CONJUNCTIVITIS CASES
}

\author{
S. JAZI, A. MOKHTARI \& A. EBRAHIMI KAHRIZSANGI \\ Department of Pathobiology, Faculty of Veterinary Medicine, \\ University of Shahrekord, Shahrekord, Iran
}

\section{Summary}

Jazi, S., A. Mokhtari \& A. Ebrahimi Kahrizsangi, 2020. Molecular detection of Chlamydia psittaci and Chlamydia felis in human keratoconjunctivitis cases. Bulg. J. Vet. Med., 23, No $1,130-137$.

Given the high incidence of keratoconjunctivitis in Iran (approximately 3.6-53.9\%) and low efficiency of clinical diagnostic measures, application of laboratory tests for detection of different keratoconjunctivitis/conjunctivitis causes and determination of their accurate prevalence is essential. In this research, conjunctival samples were collected from 100 patients with keratoconjunctivitis signs referred to an eye hospital of Iran. After DNA extraction, PCR was carried out for detection of Chlamydia psittaci and Chlamydia felis. PCR positive products were further subjected for DNA sequencing. In this study, one sample was Chlamydia psittaci positive and none was positive for Chlamydia felis. There wasn't a statistically significant relationship between working in the field of veterinary medicine or keeping a pet and Chlamydia psittaci prevalence $(\mathrm{P}>0.05)$. This study showed a low rate of chlamydial keratoconjunctivitis and therefore further studies for detection of other causes are necessary.

Key words: Chlamydia felis, Chlamydia psittaci, human, keratoconjunctivitis, PCR

Zoonotic potential of members of the genus Chlamydia is a concern, especially for human population in contact with animals. Chlamydia felis is considered an important cause of infectious diseases of the upper respiratory tract and eyes in cats, having zoonotic potential (Everson et al., 2003). A few cases of $C$. felis conjunctivitis have been documented in people. One case occurred in an HIV-infected person who suggested that he had acquired it from an infected cat. Three other cases included a renal transplant recipient having developed malaise; coughing and abnormal liver function, a person with infective endocarditis and glomerulonephritis and a healthy man who developed a febrile illness with malaise, pneumonia and mental impairment. C. felis 
nucleic acids have also been detected in respiratory samples collected from few people, including nasal swabs from a healthy person (Browning, 2004).

Avian chlamydiosis is generally called psittacosis (or ornithosis) in humans. The disease caused by $C$. psittaci in humans is called psittacosis which could be either inapparent or manifested with mild influenza-like symptoms to severe and even potentially fatal systemic disease with severe pneumonia. Psittacosis is of concern for public health authorities and specific control measures have been recommended (Aaziz et al., 2015).

Humans can be infected with any genotype. $C$. psittaci can infect people asymptomatically, cause keratoconjunctivitis or result in a systemic illness. A recent study from Egypt found C. psittaci DNA in some women presented with various gynaecological complaints (Dumke et al., 2015).

Recent studies suggest that infections with $C$. psittaci may be underdiagnosed in some populations, such as poultry workers (Krawiec et al., 2015).

Since Chlamydia psittaci can infect humans, it should be handled carefully under conditions of bio-containment. The disease is of public health significance because of the popularity of birds as pets and placement of birds in childcare facilities, garden centres and rest homes. Moreover, feral pigeons in urban habitats are commonly infected, presenting a zoonotic risk. The disease is rarely fatal in properly treated patients. Therefore, awareness of the danger and early diagnosis are important.

In Belgium, seven, two and three psittacosis cases were reported in 2005, 2006 and 2007, respectively. In Australia, Germany, Sweden and the Netherlands, 62, 10,9 and 27 cases were reported in 2007.
This is probably an underestimation as psittacosis is difficult to diagnose. Harkinezhad et al. (2009) examined the occurrence of psittacosis in a generally healthy Belgian population in contact with domestic and/or companion birds using a $C$. psittaci-specific nested PCR and serology and 69 of $540(12.7 \%)$ pharyngeal swabs were found to contain C. psittaci DNA.

Conjunctivitis is one of the commonest and highly contagious eye diseases in Iran, which frequently needs in-hospital treatment. Due to the overlap of conjunctivitis symptoms it seems very difficult to diagnose it precisely and definitely based on clinical symptoms (Mokhtari et al., 2013). Conjunctivitis diagnosis is based on history taking and physical examination but there are no key signs for etiologic diagnosis of conjunctivitis. Early symptoms of the disease are not specific and accurate distinction between viral and bacterial causes is almost impossible (Mokhtari et al., 2014).

Better diagnostic tools are needed to elucidate the role and frequency of $C$. psittaci in human disease. At present, serological tests are often used for diagnosis of $C$. psittaci infection in birds. However, serology cannot provide a definitive diagnosis due to the lack of specific antibody detection assays, the long persistence of antibody titres in cured birds and the need for convalescent sera to detect seroconversion. There is a need to validate newly developed nucleic acid amplification techniques, such as polymerase chain reaction (PCR), for rapid detection of infected animals and for diagnosis of infection in human patients (Damborg et al., 2016).

It is possible to identify chlamydial infection by culture, but results take longer than the other tests. Although culture is the legal standard, it is not the gold stan- 
dard for the detection of Chlamydia. Antigen tests are no longer recommended for Chlamydia testing due to insufficient diagnostic accuracy and PCR techniques are now the preferred option for diagnosing Chlamydia infection. Such techniques are extremely sensitive and avoid problems with poor viability of the organisms (Browning, 2004)

In previous studies reported from Iran, the prevalence of the various bacterial and viral conjunctivitis and keratoconjunctivitis varies from $3.6 \%$ to $53.9 \%$. Data about the possible involvement of Chlamydia in these complications from Iran are not available (Sohrabi et al., 2011; 2014; Mokhtari et al., 2013; 2014).

Considering the increasing prevalence of Chlamydia in abortion cases of Isfahan and Chaharmahal and Bakhtiari (neighbouring province) and the increasing intensity of contacts between humans and animals (Sohrabi et al., 2011; Damborg et al., 2016), we have designed this study as a cross-sectional survey on a target group comprising all patients referred to an ocular disease hospital with general keratoconjunctivitis symptoms. One hundred conjunctival swab samples were obtained during 2013-2014 and were analysed to detect vaccinia gene. All the procedures followed in this study were in accordance with the ethical standards and with the Helsinki Declaration of 1975, as revised in 2000. A complete history of work experience in the lab or health centres was collected. The description of samples such as sex and age groups, association with veterinary activities or pet keeping is available in Table 1. The patients' eyes were washed with sterile saline and a sterile cotton swab was rubbed onto the conjunctiva. Then it was immersed in Eagle's minimal essential medium supplemented with penicillin, amikacin, amphotericin $\mathrm{B}$ and $1 \%$ foetal bovine serum. DNA was extracted according to phenol chloroform DNA extraction protocol.

The presence of Chlamydia psittaci and Chlamydia felis was detected using the primers designed by Beacon designer software. The sequences of the forward and reverse primers were 5'-GCT ACACGCATAAGTTTACC -3' and 5'TGGAGAGAGACCTCTAGG -3 ' for C. felis and 5'- GCATTACTCGCTATA TTCC-3' and 5'-TGTGGAAGGAGA GGGAAC -3' for $C$. psittaci respectively. Each PCR reaction was performed in a final volume of $25 \mu \mathrm{L}$ containing $11 \mu \mathrm{L}$ of deionized sterile water, $10 \mu \mathrm{L}$ of Taq DNA Polymerase $2 \times$ Mix Red- $\mathrm{MgCl}_{2} 2$ mM (GeneAll, Cat. no. A180301), 1 pmol of each primer and $2 \mu \mathrm{L}$ of DNA template.

The thermal cycling conditions for the amplification were 1 cycle for $4 \mathrm{~min}$ at $94^{\circ} \mathrm{C}, 30$ cycles of $30 \mathrm{~s}$ at $94^{\circ} \mathrm{C}, 30 \mathrm{~s}$ at $53{ }^{\circ} \mathrm{C}$ for $C$. psittaci and $50{ }^{\circ} \mathrm{C}$ for $C$. felis and $25 \mathrm{~s}$ at $72{ }^{\circ} \mathrm{C}$, with a final extension step of $3 \mathrm{~min}$ at $72{ }^{\circ} \mathrm{C}$. Positive and negative controls (from Iranian Biological Resource Center) were included in each analysis. Six $\mu \mathrm{L}$ of the amplified products were loaded on a $1.3 \%$ agarose gel, and visualised by staining with ethidium bromide and compared to DNA markers (50 base pair ladder, Fermentas).

PCR positive sample in a volume of $50 \mathrm{~mL}$ was sent to Bioneer Company for sequencing. Sequencing was performed on the ABI 3730XL DNA Analyzer, with a high quality of sequence analysis data (Phred Score (QV): $\geq 20$, Guaranteed read lengths: $\geq 600 \mathrm{bp}$ ). After obtaining all results, the data was analysed using IBM SPSS 22 software.

Out of the 100 conjunctival swab samples, 1 sample (1\%) was positive for $C$. psittaci nucleotide fragment. Thirty-two 
of the cases had a history of working in the veterinary fields or keeping a pet. One out of these 32 cases were $C$. psittaci positive. Chlamydia prevalence results according to factors such as age, sex, exposure and Chlamydia positivity status are listed in Table 1. In this study there wasn't a statistically significant association between Chlamydia and working in the veterinary fields or keeping a pet (Chi square test; $\mathrm{P}>0.05$ )

Chlamydia psittaci was detected using PCR test specific for the 16s rRNA nucleotide fragment. The Chlamydia psittaci-specific band with the size of $122 \mathrm{bp}$ was detected in Chlamydia psittaci positive control. The positive PCR product was of the same size as those from the positive control. There was no Chlamydia felis positive among samples while band with the size of $159 \mathrm{bp}$ was detected in Chlamydia felis positive control (Fig. 1).

After sequencing, alignment of expected amplicon with the read sequences confirmed the presence of the 16s rRNA nucleotide fragment in the positive sample. There was $98 \%$ identity between PCR product sequences and expected ampli- con. Therefore, the result of PCR test designed for detection of Chlamydia psittaci was confirmed.

Psittacosis can cause severe serious health problems among humans. All genotypes of Chlamydia psittaci can be transmitted to humans and cause psittacosis or parrot fever. From 1988 through 2003, a total of 935 human cases of psittacosis were reported to the US Centers for Disease Control and Prevention; most were related to contact with Psittaciformes. The incidence of psittacosis in men seems to be increasing in industrialised countries and is related to importation of exotic birds. Other cases may not be correctly diagnosed or reported (Vanrompay et al., 2007).

During the 1980 s, approximately $70 \%$ of the psittacosis cases with a known source of infection resulted from human exposure to caged pet birds; of these persons, the largest group affected (43\%) included bird fanciers and owners of pet birds (Anonymous, 1996). Similarly, in the present study, one person who had positive result for Chlamydia psittaci PCR test was owner of pet birds. Human cases of psittacosis has been reported from

Table 1. The prevalence of $C$. psittaci among the studied keratoconjunctivitis patient cohort

\begin{tabular}{lccc}
\hline & C. psittaci positive & C.psittaci negative & Total \\
\hline Sex: & & & \\
female & 13 & 37 & 50 \\
male & 15 & 35 & 50 \\
\hline Age groups (years): & & & \\
$<15$ & 0 & 25 & 25 \\
$15-30$ & 1 & 24 & 25 \\
$30-45$ & 0 & 25 & 25 \\
$>45$ & 0 & 25 & 25 \\
\hline Working at veterinary & & & \\
medicine field/keeping pet: & 1 & 31 & 32 \\
yes & 0 & 68 & 68 \\
no & & & \\
\hline Exposure: & 0 & 10 & 21 \\
previous & 1 & 20 & \\
current & & & \\
\hline
\end{tabular}


many countries such as Austria, Argentina, Australia, Belgium, Bosnia and Herzegovina, Chile, Croatia, Czech Republic, Denmark, Finland, Germany, Hungary, Japan, Poland, Slovakia, Spain, Sweden, The Netherlands, UK/Great Britain, UK/N. Ireland, Ukraine, USA from 1996 to 2009 , In Iran there are reports of Chlamydia psittaci but not from keratoconjunctivitis cases (Browning, 2004).

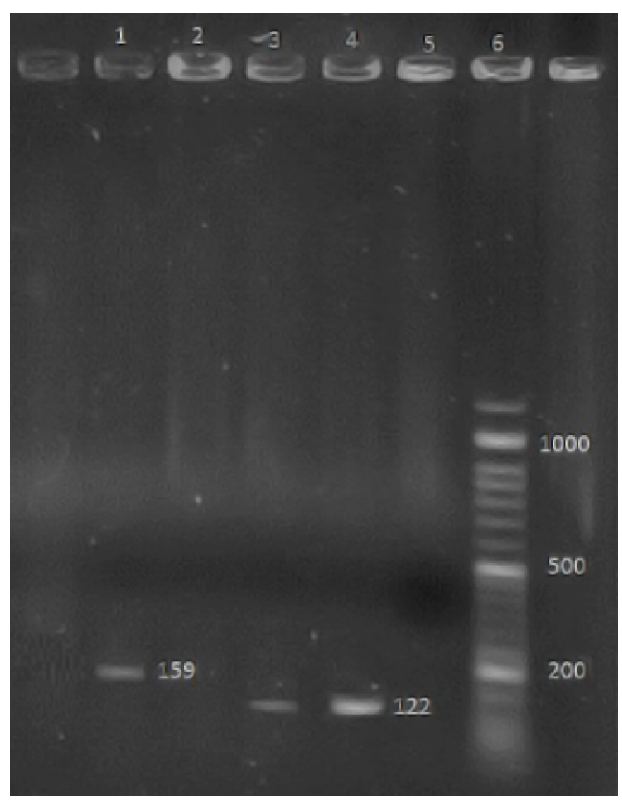

Fig. 1. C. felis and C. psittaci PCR amplification products following electrophoresis. Lane 1: C. felis positive control, lane 2: C. felis negative control, lane 3: C. psittaci positive sample, lane 4: C. psittaci positive control, lane 5: C. psittaci negative control, lane 6: 50 bp DNA ladder.

Vanrompay et al. (2007) studied zoonotic transmission of Chlamydophila psittaci in 39 breeding facilities for Psittaciformes that frequently used antimicrobial drugs. Chlamydia psittaci was detected in $14.9 \%$ of humans at these facilities (Beeckman et al., 2009). The preva- lence of Chlamydia psittaci in their research was higher than the present study which is probably due to this fact that their sampling performed from the population at the risk of transmission, but in the present study, random sampling was used.

A study in China reported that severe pneumonia in peacocks and peacock farmers was caused by $C$. psittaci (Browning, 2004). The rate of infection in the study was similar to our study; however the sampling site was different.

Harkinezhad et al. (2009) reported that the prevalence of Chlamydia psittaci infections in a human population was $12.7 \%$ using PCR and that $40.6 \%$ sera of PCR-positive individuals reacted positively in the rMOMP ELISA. Among 28 C. psittaci positive people, 24 and 4 had daily or weekly contact with domestic or companion birds, respectively (Browning, 2004).. They concluded that since previous studies were based on serological tests; psittacosis occurs more often in Belgium than reported and incidence of psittacosis is most likely also underestimated in other European countries So, in our study we used PCR test for detection of Chlamydia to have more accurate results.

Beeckman et al. (2009) showed that human psittacosis is a zoonotic infectious disease and due to a low awareness of the disease and a variable clinical presentation psittacosis is often not recognised by general practitioners. Overall, because human infection can result from brief, passing exposure to infected birds or their contaminated droppings, persons with no identified leisure-time or occupational risk can become infected (Johnston et al., 2000).

Another Chlamydia species we tried to detect was Chlamydia felis. It more com- 
monly causes conjunctivitis in cats (Halanova et al., 2011). The majority of cases are frequently associated with chronic conjunctivitis (Wills et al., 1988) and more severe ocular disease (Fernandez et al., 2016).

C. felis was suspected of causing human disease, particularly follicular conjunctivitis (Ostler et al., 1969; Schachter et al., 1969) or atypical pneumonitis (Cotton \& Partridge, 1998). Endocarditis with secondary glomerulonephritis (Regan et al., 1979) and severe liver breakdown in an immunocompromised patient (Griffiths et al., 1978) were reported. Although $C$. felis is a zoonotic pathogen, there is few epidemiological evidence for a significant zoonotic risk. Conjunctivitis caused by $C$. felis has been reported in an HIV-infected patient (Hartley et al., 2001).

Since pet cats and dogs are considered to be faithful friends and companions of humans, they play an important role in human life. Unfortunately, cats and dogs may be important sources of $C$. felis infection in humans (Aaziz et al., 2015). C. felis can induce conjunctivitis in people who are in close contact with infected cats, but the risk is extremely low, as indicated by the comparison of the seroprevalence in cats and their owners (Yan et al., 2000).

Travnicek et al. (2002) documented that Chlamydia felis has a zoonotic potential. They showed presence of $C$. felis in 13 and 10 investigated cats and human in nasal mucosa smears and conjunctiva. We couldn't found any C. felis positive sample. Given the fact that the number of samples taken from the pet owners or animal-related occupations was low in our study, and the global prevalence of $C$. felis in humans is low, this result was likely.

Browning (2004) reviewed all the reports and texts to find whether Chlamydo- phila felis is a significant zoonotic pathogen or not. He concluded that based on seven case reports $C$. felis may occasionally cause keratoconjunctivitis in humans and although at a lower rate, it can cause serious systemic disease or pneumonia.

Based on the previous studies, the likelihood of Chlamydia felis isolation from humans is low and therefore it may be considered as one of the reasons for not achieving a positive Chlamydia felis sample or a low prevalence of $C$. psittaci in this study.

Since the organism is intracellular, it is necessary to obtain good quality swabs that include cells (Segarra et al., 2011). We used PCR test for identifying two Chlamydia strains. PCR is a good test for detection of Chlamydia species. Other techniques for demonstrating the organism are less sensitive and less reliable than PCR (Streeten \& Streeten, 1985).

Harkinezhad et al. (2009) concluded clinicians should be strongly encouraged to use nucleic acid amplification tests for diagnosing Chlamydia rather than serology because molecular tests are extremely sensitive and specific and present direct evidence of a Chlamydia infection.

Although $C$. felis has a zoonotic potential, we couldn't detect it in this study. This result is in concordance with previous studies that showed that the prevalence of $C$. felis is very low. However, the failure in detection of it may be due to sampling condition, because we used only ocular swab samples. Overall, the present study showed a low rate of $C$. psittaci infection among human keratoconjunctivitis cases in Iran and no statistically significant relationship obtained between keeping pet animals and Chlamydia prevalence $(\mathrm{P}>0.05)$. Since previous studies emphasise that chlamydial zoonotic infections are rarely identified, some pre- 
caution is warranted when handling pet animals.

Further studies for determination of the prevalence of Chlamydia species in keratoconjunctivitis cases in a larger scale and consequently, preventive methods are necessary in Iran.

\section{ACKNOWLEDGEMENTS}

The authors are grateful to microbiology laboratory personnel of Faculty of Veterinary Medicine, University of Shahrekord for their collaboration.

\section{REFERENCES}

Ahmadi, M. H., A., Mirsalehian \& A. Bahador, 2015. Prevalence of genital Chlamydia trachomatis in Iran: A systematic review and meta-analysis. Pathogens and Global Health, 109, 290-299.

Aaziz, R., P., Gourlay, F. Vorimore, K. Sachse, V. I. Siarkou \& K. Laroucau, 2015. Chlamydiaceae in North Atlantic seabirds admitted to a wildlife rescue center in Western France. Applied and Environmental Microbiology, 81, 4581-4590.

Anonymous, 1996. Compendium of measures to control Chlamydia psittaci infection among humans (psittacosis) and pet birds (avian chlamydiosis), 1998. Morbidity and Mortality Weekly Report, 47 (RR10), 1-9.

Beeckman, D. S. \& D. C. Vanrompay, 2009. Zoonotic Chlamydophila psittaci infections from a clinical perspective. Clinical Microbiology and Infection, 15, 7-11.

Browning, G. F., 2004. Is Chlamydophila felis a significant zoonotic pathogen? Australian Veterinary Journal, 82, 695-596.

Damborg, P., E. M. Broens, B. B. Chomel, S. Guenther, F. Pasmans, J. A. Wagenaar, J. S. Weese, L. H. Wieler, U. Windahl, D. Vanrompay \& L. Guardabassi, 2016. Bacterial zoonoses transmitted by household pets: State-of-the-art and future perspectives for targeted research and policy ac- tions. Journal of Comparative Pathology, 155, S27-S40.

Fernandez, M., E. G. Manzanilla, A. Lloret, M. León \& J. C. Thibault, 2016. Prevalence of feline herpesvirus-1, feline calicivirus, Chlamydophila felis and Mycoplasma felis DNA and associated risk factors in cats in Spain with upper respiratory tract disease, conjunctivitis and/or gingivostomatitis. Journal of Feline Medicine and Surgery, 19, 461-469.

Gaydos, C. A., L. Palmer, T. C. Quinn, S. Falkow \& J. J. Eiden, 1993. Phylogenetic relationship of Chlamydia pneumoniae to Chlamydia psittaci and Chlamydia trachomatis as determined by analysis of $16 \mathrm{~S}$ ribosomal DNA sequences. International journal of Systematic Bacteriology, 43, 610-612.

Halanova, M., Z. Sulinova, L. Cislakova, A. Trbolová, L. Páleník, T. Weissová, M. Halán, Z. Kalinová \& M. Holičková, 2011. Chlamydophila felis in cats - are the stray cats dangerous source of infection? Zoonoses and Public Health, 58, 519-522.

Harkinezhad, T., K. Verminnen, M. De Buyzere, E. Rietzschel, S. Bekaert \& D. Vanrompay, 2009. Prevalence of Chlamydophila psittaci infections in a human population in contact with domestic and companion birds. Journal of Medical Microbiology, 58, 1207-1212.

Hartley, J. C., S. Stevenson, A. J. Robinson, J. D. Littlewood, C. Carder, J. Cartledge, C. Clark \& G. L. Ridgway, 2001. Conjunctivitis due to Chlamydia felis (Chlamydia psittaci feline pneumonitis agent) acquired from a cat: Case report with molecular characterization of isolates from the patient and cat. Journal of Infection, 43, 711.

Hashemi, F. B., B. Pourakbari \& J. Zaeimi Yazdi, 2007. Frequency of Chlamydia trachomatis in Women with Cervicitis in Tehran, Iran, Infectious Diseases in Obstetrics and Gynecology, 2007, 67014-67018.

Johnston, W. B., M. Eidson \& M. G. Stobierski, 2000. Compendium of measures to 
control Chlamydia psittaci infection among humans (psittacosis) and pet birds (avian chlamydiosis). Morbidity and Mortality Weekly Report, 49, 1-17.

Meyer, T., 2016. Diagnostic procedures to detect Chlamydia trachomatis infections. Microorganisms, 4, 25.

Mokhtari, M., A. R. Laripour Tehranfar, S. Moghim, A. R. Dehghani, Z. Farajzadegan \& N. Hosseini, 2013. Determination of prevalence, sensitivity and specificity of clinical signs and symptoms of adenoviral keratoconjunctivitis. Journal of Isfahan Medical School, 30, 2029-2038.

Mokhtari, M., K. Vosta, T. Narimani, N. Hosseini \& Sh. Moghim, 2014. Prevalence of herpetic keratitis in patients referred to Feiz Ophthalmology Center, Isfahan, Iran, using polymerase chain reaction method. Journal of Isfahan Medical School, 32, 321-329.

Segarra, S., K. Papasouliotis \& C. Helps, 2011. The in vitro effects of proxymetacaine, fluorescein, and fusidic acid on realtime PCR assays used for the diagnosis of Feline herpesvirus 1 and Chlamydophila felis infections. Veterinary Ophthalmo$\log y, 14,5-8$.

Sohrabi, M. \& Z. Goodarzi, 2014. The prevalence of viral conjunctivitis in patients who referred to eye specialist hospitals in Tehran, Iran. Iranian Journal of Ophthalmology, 26, 29-32.

Sohrabi, N., M. Tebianian \& H. Moeini, 2011. Analyzing bacterial agents of keratoconjunctivitis in patients referred to Ophthalmology Ward of Feiz Hospital in Isfahan. Journal of Fasa University of Medical Sciences, 2, 95-100.
Streeten, B. W. \& E. A. Streeten, 1985. "Bluebody" epithelial cell inclusions in conjunctivitis. Ophthalmology, 92, 575-579.

Trávnicek, M., S. Mardzinová, L. Cisláková, I. Valocký \& T. Weissová, 2002. Chlamydial infection of cats and human health. Folia Microbiologica, 47, 441-444.

Vanrompay, D., T. Harkinezhad, M. van de Walle, D. Beeckman, D. van Droogenbroeck, K. Verminnen, R. Leten, A. Martel \& K. Cauwerts, 2007. Chlamydophila psittaci transmission from pet birds to humans. Emerging Infectious Diseases, 13, $1108-1110$

Wills, J. M., P. E. Howard, T. J. GruffyddJones \& C. M. Wathes, 1988. Prevalence of Chlamydia psittaci in different cat populations in Britain. Journal of Small Animal Practice, 29, 337-339.

Paper received 15.12.2017; accepted for publication 16.03.2018

\section{Correspondence:}

A. Mokhtari

Department of Pathobiology,

Faculty of Veterinary Medicine,

University of Shahrekord,

P.O Box 115, Shahrekord, Iran,

Tel: +983832324427,

Fax: +9832324427,

email: a.mokhtari@alumni.ut.ac.ir 